\section{Knowledgebase and Lifestyle Choices in Patients with Psoriatic Arthritis}

\section{To the Editor:}

Psoriatic arthritis (PsA) is a chronic inflammatory disease involving the skin and joints. It requires detection in primary care, subsequent referral, and initiation of management in secondary care and ongoing joint management. PsA has been associated with greater cardiovascular (CV) disease mortality, morbidity, and atherosclerosis ${ }^{1}$. The increased CV mortality risk has been shown to be comparable with that of rheumatoid arthritis (RA) and diabetes (risk ratio $1.74,95 \% \mathrm{CI} 1.32-2.30)^{2}$. In addition, risk factors for $\mathrm{CV}$ disease, such as hypercholesterolemia, impaired glucose tolerance, and obesity, have all been associated with PsA ${ }^{1,2,3}$.

We sought to establish the current knowledgebase and lifestyle choices of patients with PsA managed in secondary care. This data could then guide changes to services to assist patients in reducing their risk of CV morbidity and mortality.

A hundred patients diagnosed with PsA sequentially attending a rheumatology outpatient clinic completed an anonymous electronic questionnaire. Patients were asked to fill in this questionnaire in the clinic waiting area on a tablet device or a desktop computer. We enquired about current lifestyle choices, including smoking, alcohol intake, and whether they followed a low-fat diet. In addition, we asked about their awareness of associated health risks and the desire for an annual health assessment. There was also the option for a free text response regarding suggested improvements to the service and their treatment. The questionnaire took a patient about 5 minutes to complete.

Fifty-seven percent of patients were unaware of any additional health risks they faced as a patient with PsA (Figure 1) and 87\% said they would appreciate an annual health assessment to screen for diabetes, hypertension, and hypercholesterolemia. Sixteen percent of the questioned patients with PsA smoked, which is less than the UK national average of $20 \%$ in $2012^{4}$. Of these 16 patients who smoked, 14 had considered cessation and 5 wished to be referred to a rheumatology-based smoking cessation service. One percent of the group admitted to a weekly alcohol consumption of over 20 units with $87 \%$ consuming 10 units or less. Sixty-nine percent consciously tried to follow a low-fat diet. Interestingly, those patients who were aware of associated health risks were more likely to be nonsmokers and consciously followed a low-fat diet ( $\mathrm{p}$ values 0.3 and 0.31 , respectively).

One of the top 3 unmet patient needs evident in the free text responses was access to information on PsA, advice on lifestyle choices, and information related to associated PsA health risks (mentioned in 7 responses).

It is evident that only a minority of patients were aware of additional health risks associated with PsA, highlighting a need for improvement in patient education and access to information about PsA. Our work has also suggested that patient awareness of health risks was associated with healthier lifestyle choices. An annual health assessment, which is not currently routine practice for those with PsA as it is for RA, could improve personal and public health outcomes.

Given that the current CV risk incurred from PsA has been deemed to be equal to that of $\mathrm{RA}^{2}$, there is a rationale behind its inclusion in widely used primary care tools such as the QRISK2 score ${ }^{5}$. Consideration of CV risk screening for patients with PsA in primary care has been recommended $^{2,6}$. Given that in the United Kingdom CV risk scoring and intervention are managed in primary care, general practitioners (GP) would be best placed to assess the $\mathrm{CV}$ risk and arrange relevant screening.

It has been demonstrated that there is a need for better education and involvement of patients with PsA in their care ${ }^{7}$. Information regarding the additional health risks could be imparted verbally, in written form, or through online sources. Lifestyle advice should be tailored to the individual with an emphasis on sustainable changes ${ }^{8}$. To best facilitate lifestyle changes, it may be helpful for information to be imparted both in the primary and secondary care settings; however, followup on lifestyle interventions would be most accessible in primary care.

Smoking contributes to the absolute $\mathrm{CV}$ risk $^{7}$, and cessation should be promoted in both primary care and secondary care. A smoking cessation service is likely to be more readily accessed by patients if provided through primary care. Additional resources and time spent invested in educating patients with PsA could decrease longterm morbidity and mortality.

Further exploration into the proportion of GP that currently consider the $\mathrm{CV}$ risk in patients with PsA would be interesting, given that $\mathrm{CV}$ risk screening generally takes place in primary care. A joint approach between the primary care physician and the rheumatologist in educating and screening patients with PsA could help improve knowledgebase and CV health of this at-risk population.

ANNA TIMMIS, MBBS, BSc, Department of Primary Care and Public Health, Imperial College London, London, UK; MORAG LENMAN, MBBS, PhD, Imperial General Practice (GP) Specialty Training, Department of Primary Care and Public Health, Imperial College London, London, UK; SIMONE CASTAGNO, ACGI, BEng, Department of Medicine, Imperial College London, London, UK; JONAS MMESI, RN, Diploma in General Nursing, Midwifery and Psychiatry, and Diploma in Programme and Project Management, Department of Rheumatology, Imperial College Healthcare National Health Service (NHS) Trust, London, UK; DILRUKSHI TENNEKONE, MBBS, MD, Department of Rheumatology,

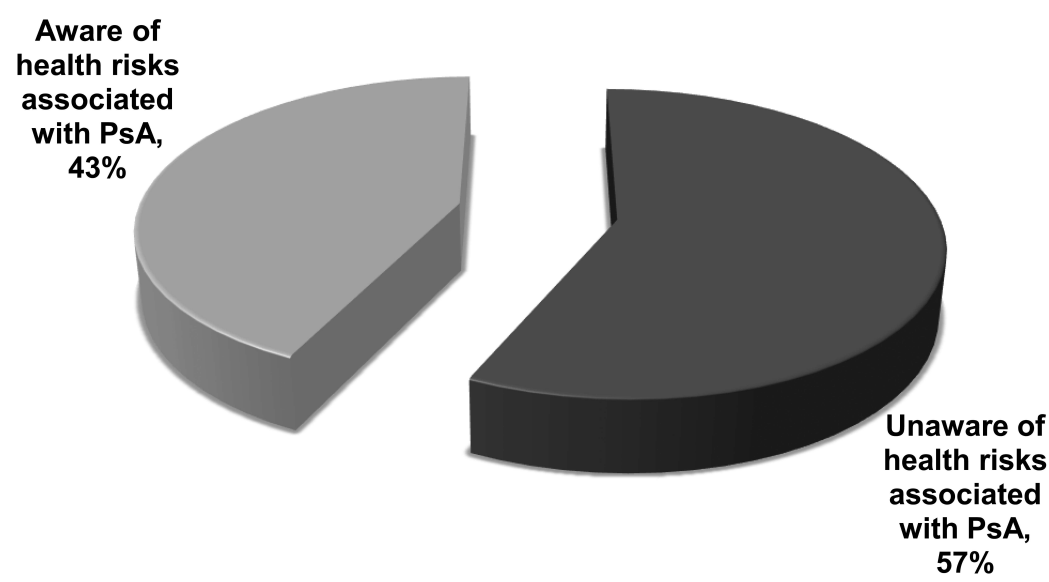

Figure 1. Pie chart showing the proportion of patients who were unaware of any additional health risks associated with PsA. PsA: psoriatic arthritis. 
Imperial College Healthcare NHS Trust, London, UK; SONYA ABRAHAM, PhD, FRCP, FHEA, Department of Rheumatology, Imperial College Healthcare NHS Trust, London, UK. Address correspondence to Dr. Anna Timmis, Imperial GP Specialty Training, Department of Primary Care and Public Health, Imperial College London, Charing Cross Campus, Reynolds Building, London W6 8RP, UK.

E-mail: anna.timmis@doctors.org.uk

\section{REFERENCES}

1. Jamnitski A, Symmons D, Peters MJ, Sattar N, McInnes I, Nurmohamed MT. Cardiovascular comorbidities in patients with psoriatic arthritis: a systematic review. Ann Rheum Dis 2013;72:211-6

2. Johnsson H, McInnes IB, Sattar N. Cardiovascular and metabolic risks in psoriasis and psoriatic arthritis: pragmatic clinical management based on available evidence. Ann Rheum Dis 2012;71:480-3.

3. Dubreuil M, Rho YH, Man A, Zhu Y, Zhang Y, Love TJ, et al. Diabetes incidence in psoriatic arthritis, psoriasis and rheumatoid arthritis: a UK population-based cohort study. Rheumatology 2014;53:346-52.
4. Health \& Social Care Information Centre. Statistics on Smoking England, 2012. [Internet. Accessed September 23, 2015.] Available from: www.hscic.gov.uk/catalogue/PUB07019

5. Collins GS, Altman DG. Predicting the 10 year risk of cardiovascular disease in the United Kingdom: independent and external validation of an updated version of QRISK2. BMJ 2012;344:e4181.

6. Peters MJ, Symmons DP, McCarey D, Dijkmans BA, Nicola P, Kvien TK, et al. EULAR evidence-based recommendations for cardiovascular risk management in patients with rheumatoid arthritis and other forms of inflammatory arthritis. Ann Rheum Dis 2010;69:325-31.

7. Leung YY, Tam LS, Lee KW, Leung MH, Kun EW, Li EK. Involvement, satisfaction and unmet health care needs in patients with psoriatic arthritis. Rheumatology 2009;48:53-6.

8. Drăgoi RG, Ndosi M, Sadlonova M, Hill J, Duer M, Graninger W, et al. Patient education, disease activity and physical function: can we be more targeted? A cross sectional study among people with rheumatoid arthritis, psoriatic arthritis and hand osteoarthritis. Arthritis Res Ther 2013;15:R156.

J Rheumatol 2016;43:1; doi:10.3899/jrheum.150335 